\title{
Unilateral Wakeup for Mobile Ad Hoc Networks with Group Mobility
}

\author{
Shan-Hung Wu, Jang-Ping Sheu, Fellow, IEEE, and Chung-Ta King
}

\begin{abstract}
Asynchronous wakeup schemes have been proposed for ad hoc networks to increase the energy efficiency of wireless communication. The basic idea is to allow a node to sleep when it is idle, and wakeup periodically to check if there are pending transmissions. In this paper, we examine the applicability of asynchronous wakeup schemes to the Mobile Ad Hoc NETworks (MANETs). We discover that, although it is desirable to have nodes with lower mobility to sleep more in reaction to the less-changing link states, in practice this is prohibited due to an unwanted tradeoff between the energy saving and in-time link discovery. All nodes in a network must stay awake frequently based on their highest possible moving speed to avoid network partition. To address this problem, we propose a new wakeup scheme, named Unilateral- (Uni-) scheme, for MANETs that allows nodes with slower moving speed to sleep more without losing the network connectivity. The Uni-scheme supports both the entity mobility and group mobility of nodes, thus has broad applicability. Theoretical analysis and simulation are conducted and show that the Uni-scheme can render significant energy saving as compared with the previous arts.
\end{abstract}

Index Terms-Mobile ad hoc networks, energy conservation, quorum systems, unilateral wakeup, group mobility

\section{INTRODUCTION}

$\mathrm{M}$ OBILE Ad Hoc NETworks (MANETs) are self-organizing networks that allow mobile nodes (or stations) to communicate with each other in the situations, such as battlefield commanding, disaster area probing, road traffic monitoring, and wildlife conservation, where centralized control is costly or infeasible. One design goal of MANETs is to ensure the energy efficiency of wireless communication to prolong the network lifetime. At PHY layer, when a node is not transmitting, the transceiver persists in idle mode and continuously listens for incoming transmissions. Studies [16], [23] report that the energy consumed by a wireless module in listening to the network is only slightly lower than that of transmitting and receiving data. If there are seldom transmissions destined to the station, idle listening would waste significant amount of energy. To address this problem, the concept of asynchronous wakeup is introduced at MAC layer, which, instead of idle listening, allows a station to sleep (or doze)—to suspend the transceiver-when there is no data transmission.

The merit of asynchronous wakeup is that stations can decide when to sleep in a distributed manner while being able to communicate with each other during the awake periods. Specifically, the time axis on each station is divided evenly into beacon intervals. A station may stay either awake or sleep during each beacon interval. By adopting a wakeup scheme [11], [20], [32], [33], [34], [35], [37] and choosing an integer $n$, the station obtains a cycle pattern, which specifies the awake/sleep schedule during $n$ continuous beacon

- The authors are with the Department of Computer Science, National Tsing Hua University, No. 101, Section 2, Kuang-Fu Road, Hsinchu, Taiwan 30013. E-mail: \{shwu, sheujp, king\}@cs.nthu.edu.tw.

Manuscript received 7 Nov. 2011; revised 26 June 2012; accepted 12 Oct. 2012; published online 28 Nov. 2012.

For information on obtaining reprints of this article, please send e-mail to: tmc@computer.org, and reference IEEECS Log Number TMC-2011-11-0597. Digital Object Identifier no. 10.1109/TMC.2012.243. intervals. The station repeats the schedule every $n$ beacon intervals, and $n$ is called the cycle length. The wakeup scheme ensures that at least one of the awake beacon intervals on a station must overlap that on another station, even when the stations' clocks (used to divide beacon intervals) are not synchronized. By exchanging the awake/ sleep schedules during the overlapped beacon interval, neighbor stations can discover each other, i.e., to know each other's wake up time, and begin data communication then.

In this paper, we examine the applicability of asynchronous wakeup to the MANETs. We discover that while it is desirable to have nodes with lower mobility to sleep more in reaction to the less-changing link states; however, in practice this is prohibited by most existing wakeup schemes [11], [20], [32], [33], [34], [35], [37] due to an unwanted tradeoff between the energy saving and in-time link discovery. Basically, an asynchronous wakeup scheme requires a station with cycle length $n$ to remain awake at least $O(\sqrt{n})$ beacon intervals per cycle to ensure the overlap [20]. The longer the cycle length, the more the power saving (PS). Nevertheless, two adjacent stations adopting cycle lengths $m$ and $n$, respectively, can only discover each other after a delay of $O(\max (m, n))$ beacon intervals. If these two nodes have high relative moving speed, the values of $m$ and $n$ must be both small to ensure the in-time neighbor discovery. Since there is no way for a station to measure its relative speed to another before the neighbor discovery (and signal exchange), the $O(\max (m, n))$ delay implies that all nodes in a network must conservatively pick a small cycle length corresponding to their highest possible relative speed to ensure the network connectivity. Given the $O(\sqrt{b})$ bound, the power saving effect can be severely restricted.

To address this problem, we propose a novel wakeup scheme, named the Unilateral- (Uni-) scheme, for MANETs that allows a node with slower moving speed to save energy by choosing a longer cycle length unilaterally regardless of its relative speed to the others. Specifically, the Uni-scheme guarantees that two adjacent stations adopting 
cycle lengths $m$ and $n$, respectively, can discover each other within $O(\min (m, n))$ beacon intervals. It is sufficient for any of these two nodes to pick a small cycle length to ensure the in-time neighbor discovery. By requiring a faster moving station to have a shorter cycle length and a slower to have a longer, we show that all nodes in a network can obtain cycle lengths corresponding to their individual speed rather than the highest possible relative one. As ordinary nodes (e.g., soldiers walking on a battlefield) usually move way slower than the fastest one (e.g., soldiers carried by an armored vehicle), this extends the cycle lengths on the majority of nodes, and saves the overall energy consumption.

To the best of our knowledge, the Uni-scheme is the first wakeup scheme that is able to give the $O(\min (m, n))$ neighbor discovery delay and allow a network to save energy by taking advantages of the nodes' diverse mobility. The design of Uni-scheme also takes into account the mobility patterns of nodes. We show that the Uni-scheme can be readily applied to the environments where either the entity mobility or group mobility [6] is presented. In the situations where nodes move in groups, the Uni-scheme can be extended to ensure that: 1) nodes from different groups, which usually have high relative speed, can discover each other in-time; 2) to save energy, nodes in the same group can pick their cycle lengths corresponding to the highest relative speed within the group rather than that between different groups. As the intragroup mobility is usually much lower than the intergroup mobility [3], [17], [30], the cycle lengths can be further prolonged. A rigorous theoretical analysis and extensive simulations are conducted. Experimental results show that the Uni-scheme is able to render more than 11 and 34 percent improvement in energy efficiency for the environments with entity and group mobility, respectively.

The rest of this paper is organized as follows: In Section 2, we review existing wakeup schemes at MAC layer. Section 3 looks into some practical limitations in MANETs and introduces the Uni-scheme. The overlap guarantees given by the Uni-scheme are formally proven in Section 4. In Section 5, we extend the Uni-scheme and its fundamentals for environments with group mobility. The performance evaluation is conducted in Section 6. Finally, Section 7 drops the conclusions.

\section{Preliminaries}

In this section, we describe our target environments and review existing wakeup schemes. Some terminologies and assumptions are specified as well that will be used throughout the text.

\subsection{Target Environments}

We focus on MANETs with either the entity mobility, where nodes move independently in different directions and speed, or group mobility, where some nodes' decisions on directions and speed depend upon the others in the same group [6]. We assume that a node is aware of its own moving speed by either a speedometer (ultrasonic-, infrared-, inductive loop-, or vision-based), GPS receiver, or triangulation ${ }^{1}$ of the signal strengths from nearby nodes [2], [13], [14].

1. A simpler two-node model has also been proposed by the PATH [14] at UC Berkeley.

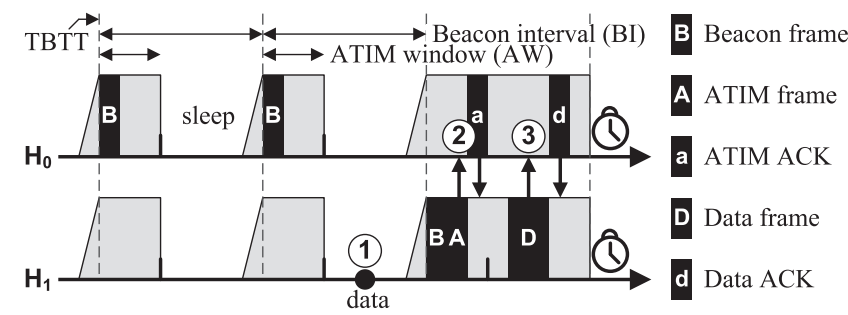

Fig. 1. The structure of awake/sleep beacon intervals in IEEE 802.11 Power Saving mode.

In presence of group mobility, nodes in the same group usually have relative speed slow enough allowing one or more clusters to be formed [3], [15], [17], [26], [28], [30]. In each cluster, a node (usually having the slowest aggregated relative speed [3] with its neighbors) is delegated as the clusterhead to coordinate communications between members (i.e., ordinary nodes). Nodes at the border of a cluster become relays to forward data between clusters. As compared with the flat structure, the clustered network topology improves both the scalability and energy efficiency due to the localization of node dynamics [8], [19], reuse of resources (such as bandwidth and codes) [18], [26], and simplification of routing paths [31]. Note the proposed Uni-scheme at MAC layer is transparent to the routing/ clustering algorithms at network/application layers, and can be applied to either the flat or clustered environments.

\subsection{Related Work}

IEEE 802.11 Power Saving Mode. The operation of IEEE 802.11 Power Saving mode [12] is shown in Fig. 1. On each station, the time axis is divided evenly into beacon intervals. In every beacon interval, the station is required to remain awake during the entire Announcement Traffic Indication Message (ATIM) window. A beacon frame is broadcasted at the Target Beacon Transmission Time (TBTT) to announce the station's existence. If a station, say $H_{1}$, intends to transmit data to a destination $H_{0}$ (Fig. 1(1)), it first unicasts an ATIM frame to $H_{0}$ during the ATIM window (Fig. 1(2)). Remaining awake, $H_{0}$ receives the ATIM frame and sends back an acknowledgment. Both $H_{0}$ and $H_{1}$, after this ATIM notification procedure, keep awake for the entire beacon interval and start data transmission after the end of ATIM window (Fig. 1(3)). To avoid data collisions, the data transmission follows the RTS, CTS, and random back-off procedure specified in the Distributed Coordination Function (DCF). ${ }^{2}$ If there is no ATIM notifications, stations may enter the doze mode (that is, to sleep) to save energy after each ATIM window. We denote the duration of a beacon interval and an ATIM window as $\bar{B}$ and $\bar{A}$, respectively.

The IEEE 802.11 PS mode functions only when the timers on stations are synchronized (or equivalently, when the TBTT is aligned). As synchronizing the clocks in MANETs is usually costly or even infeasible [5], there is a need for an energy conservation protocol that admits the asynchronous timers between stations.

2. In the situation where data transmission cannot complete within a single beacon interval (due to collisions or large data volume), $H_{0}$ can set the more-data bit (in data frame header) true telling $H_{1}$ to remain awake through the successive beacon interval to continue data transmission [12]. 


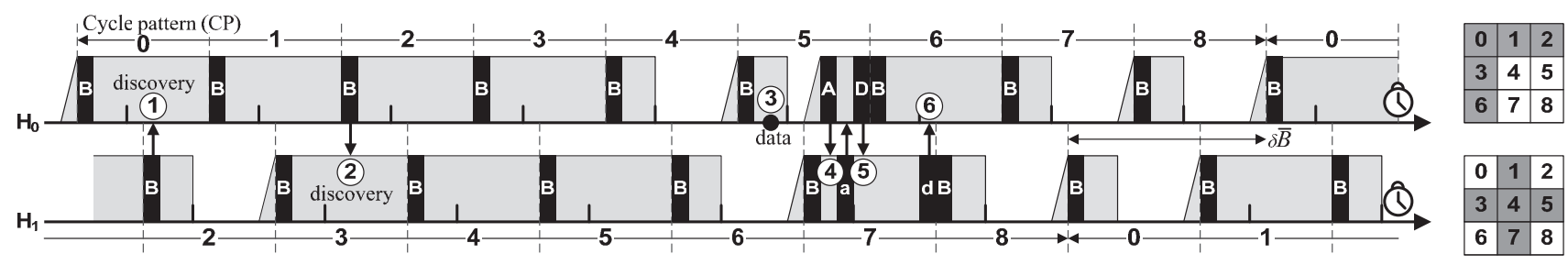

Fig. 2. Asynchronous wakeup based on the grid quorum scheme. Stations with arbitrary timer shift $\delta \bar{B}$ are guaranteed to discover each other. In this case, both $H_{0}$ and $H_{1}$ choose the same cycle length $n=9$.

Asynchronous Wakeup Protocols. Based on the IEEE 802.11 PS mode, the idea of asynchronous wakeup protocols, also known as the Asynchronous Quorum-based Power Saving (AQPS) protocols, is to prolong stations' awake periods at certain beacon intervals to ensure the beacon exchanges. Given a cycle length denoted by $n$, an AQPS protocol numbers $n$ continuous beacon intervals from 0 to $n-1$, and defines a quorum, a subset of $\{0,1, \ldots, n-1\}$, for each station. The set of quorums is named a quorum system. During those beacon intervals whose numbers are specified in the quorum, a station must remain awake after the ATIM window, even when there is no data transmission. This awake/sleep schedule repeats every $n$ beacon intervals and is called the cycle pattern. Fig. 2 gives an example where two stations adopt quorums $\{0,1,2,3,6\}$ and $\{1,3,4,5,7\}$, respectively, to form their cycle patterns. It can be shown [20], [35] that two adjacent stations with arbitrary timer shift $\delta \bar{B}, \delta \in \mathbb{R}$, are guaranteed to exchange their beacons within a finite delay if their quorums intersect. ${ }^{3}$ Note that in an AQPS protocol the beacon frames carry additional information about the awake/sleep schedule of the sending station, such as the selected quorum and the number of current beacon interval, and so on. Once receiving a beacon (Figs. 2(1) and 2(2)), a station can discover the sending party and predict its next awake period. The ATIM notification and data transmission procedures can start thereafter when data arrive (Figs. 2(3), 2(4), 2(5), and 2(6)).

A quorum system can be constructed using different quorum schemes (also called the wakeup schemes), such as grid/torus [7], [20], [32], [35], finite projective plane [11], or difference set [9], [33], [34]. In the following, we briefly summarize the grid/torus scheme as it is relevant to our study. By assuming that $n$ is a square, a grid scheme organizes the numbers $0,1, \ldots, n-1$ as an $\sqrt{n} \times \sqrt{n}$ array in a row-major manner, as shown in Fig. 2. It defines a quorum as a set containing all numbers along a column and a number from each of the remaining columns (e.g., $\{0,1,2,3,6\}$ and $\{1,3,4,5,7\}$ adopted by $H_{0}$ and $H_{1}$, respectively). By definition, we can easily see that any two quorums intersect. This ensures the neighbor discovery between every pair of nodes in a network. ${ }^{4}$ Note a quorum defined by the grid-scheme has quorum size (i.e., cardinality) $2 \sqrt{n}-1$. Study [20] shows that a quorum applicable to an AQPS protocol can have size no smaller than $\sqrt{n}$. The larger the cycle length $n$, the more the power saving.

3. Not every quorum system is applicable to an AQPS protocol. The quorums system must be cyclic to ensure the neighbor discovery when the timers shift between stations. The formal definition of the cyclic property will be given in Section 4 .

4. A quorum system constructed by the grid/torus scheme is cyclic.
Other combinatorics, such as the finite projective plane [11] and difference set [9], can be used to construct a quorum system. Ideally, quorums defined by the finite projective plane can have smaller quorum sizes than the grid/torus-based quorums. However, currently these quorums need to be searched exhaustively [11] to form a quorum system, which can be very time consuming. On the other hand, quorums composed in [9] usually have large quorum sizes close to $n$ when $n$ is small. This limits the energy saving effect severely, as in MANETs $n$ is usually forced to be small to ensure bound delay (such that, for example, the route advertisements at Network layer can be sent properly). Therefore, this scheme [9] is only suitable for delay-tolerant networks.

Recently, the grid-scheme is extended [7], [10], [34] to allow the nodes to pick different cycle lengths, as shown in Fig. 3a, without losing the network connectivity. The motivation is that the delay incurred by an AQPS protocol is proportional to $n$, so by picking different cycle lengths dynamically, a node can control the tradeoff between energy efficiency and delay based on its own current needs (such as the remaining battery life, traffic type, and traffic load, etc.).

Another extension [25], [33], [35], which targets the clustered networks, releases the overlap requirement between the quorums of members. By relying on the clusterheads/relays to forward the members' existence, there is no need to insist the neighbor discovery between members. As shown in Fig. 3b, each member with this relaxed overlap requirement can simply pick a set containing all numbers along a column as its quorum. The member is guaranteed to discover its clusterhead/relays, but not the other members. Quorums of this type have size $\sqrt{n}$, which is only nearly half of those of the clusterheads/relays, so the energy efficiency of members can be improved. Note that

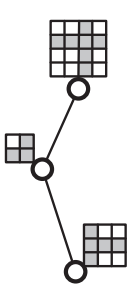

(a)

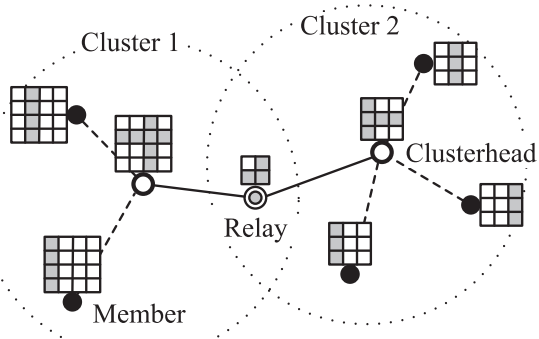

(b)
Fig. 3. Extensions of the grid-based wakeup scheme. (a) Nodes choosing different cycle lengths are guaranteed to discover each other. (b) Members in clustered networks can use a new type of quorums with smaller sizes. 
while clusterheads/relays can freely adapt their cycle lengths, the cycle lengths chosen by members must be identical to the ones decided by their clusterheads.

In this paper, we propose the notion of unilateral wakeup and a new quorum scheme, called the Uni-scheme, to address some limitations of existing AQPS protocols when applied to MANETs. Note that our preliminary results have been published as a conference version [36]. However, this version did not consider the situation where nodes move in groups. As group mobility is one of the most prominent characteristics of MANETs and is a main factor affecting link discovery, route establishment, clustering, and thereby power consumption, in this paper we study how the notion of unilateral wakeup can be extended to be applied to MANETs with group mobility, in Section 5, and to further improve energy efficiency. Specifically, we 1) extend the proposed Uni-scheme to allow nodes to take advantages of slow relative speed within a moving group to save energy; and 2) rigorously proof the overlap guarantee given by the extended Uni-scheme to ensure network connectivity. We also extend the section for performance evaluation (Section 6) to consider different intragroup speed in presence of group mobility in theoretical analysis, and to compare the Uni-schme with existing wakeup schemes under various network conditions in simulations.

\section{UNILATERAL WAKEUP FOR MANETs}

While many schemes are proposed, little effort has been made to understand the impact of asynchronous wakeup to the MANETs. In this section, we first look into the delays incurred by existing wakeup schemes. Then, we propose the Uni-scheme to address some practical limitations.

\subsection{Limitation of Existing AQPS Protocols}

The power saving advantage given by an AQPS protocol comes at the price of delay. This includes the neighbor discovery delay, i.e., the time required for a station to discover its new neighbor, and data buffering delay, i.e., the duration between a packet arrival (on a sending station) and its start of DCF. Since a receiving station must remain awake during the ATIM window of each beacon interval, the data buffering delay can be no longer than a $\bar{B}$ (usually $100 \mathrm{~ms}$ [12]). On the other hand, two adjacent nodes picking the cycle lengths $m$ and $n$, respectively, to form their quorums $Q(m)$ and $Q(n)$ following the existing wakeup schemes [7], [9], [34], [35] can only discover each other after a worst-case delay $l_{Q(m), Q(n)}=(\max (m, n)+$ $\min (\sqrt{m}, \sqrt{n})) \bar{B}=O(\max (m, n)) \bar{B}$. The larger the cycle lengths, the longer the neighbor discovery delay. Note we have $l_{Q(m), Q(n)} \leq \max \left(l_{Q(m), Q(m)}, l_{Q(n), Q(n)}\right)$.

The impact of asynchronous wakeup to a mobile node $H_{0}$ can be characterized as a zone of uncertainty, as shown in Fig. 4. In this area, neighbors of $H_{0}$ may not be discovered (Fig. 4(1)) unless they move into the discovery zone, where the neighbor discovery is guaranteed. Denote $r$ and $d$ the radiuses of node coverage and discovery zone, respectively. To make the impact transparent to the upper layers (e.g., routing protocols), one may regard the discovery zone as the effective node coverage. Like $r$, the proper value of $d$ depends on the application needs. Given $r$ and $d$, the

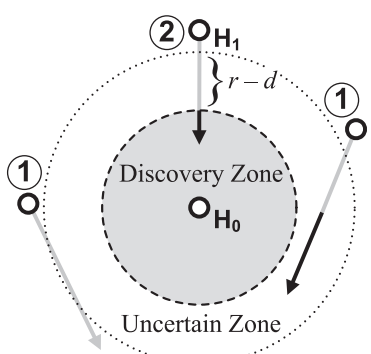

Fig. 4. The asynchronous wakeup divides the coverage of a node into the zones of discovery and uncertainty. The node $H_{0}$ moves upwards and the relative moving trajectories of its neighbors are depicted as solid lines. A trajectory becomes black if the neighbor is discovered.

node $H_{0}$ must ensure that, for each neighbor $H_{1}$, it is able to discover $H_{1}$ before $H_{1}$ enters the discovery zone, i.e.,

$$
\left(s_{0}+s_{1}\right) \cdot l_{Q\left(n_{0}\right), Q\left(n_{1}\right)} \leq r-d,
$$

where $s_{i}$ and $n_{i}$ denote the moving speed and cycle length of $H_{i}$, respectively, and $s_{0}+s_{1}$ is the highest (face to face) relative speed between $H_{0}$ and $H_{1}$, as shown in Fig. 4(2). The picked $n_{0}$ and $n_{1}$ must satisfy

$$
l_{Q\left(n_{0}\right), Q\left(n_{1}\right)} \leq \frac{r-d}{s_{0}+s_{1}} .
$$

However, $n_{1}$ and $s_{1}$ are unknown to $H_{0}$ (so are $n_{0}$ and $s_{0}$ to $H_{1}$ ). To discover each other in-time, both of the two nodes must pick a conservative $n_{i}$ such that

$$
l_{Q\left(n_{i}\right), Q\left(n_{i}\right)} \leq \frac{r-d}{s_{i}+s_{\text {high }}},
$$

where $s_{\text {high }}$ is the highest possible moving speed of a node, as

$$
\begin{aligned}
l_{Q\left(n_{0}\right), Q\left(n_{1}\right)} & \leq \max \left(l_{Q\left(n_{0}\right), Q\left(n_{0}\right)}, l_{Q\left(n_{1}\right), Q\left(n_{1}\right)}\right) \\
& \leq \frac{r-d}{\min \left(s_{0}, s_{1}\right)+s_{\text {high }}} \leq \frac{r-d}{s_{0}+s_{1}} .
\end{aligned}
$$

Note $l_{Q\left(n_{0}\right), Q\left(n_{1}\right)} \leq \max \left(l_{Q\left(n_{0}\right), Q\left(n_{0}\right)}, l_{Q\left(n_{1}\right), Q\left(n_{1}\right)}\right)$ follows from the fact that $l_{Q\left(n_{0}\right), Q\left(n_{1}\right)}=\left(\max \left(n_{0}, n_{1}\right)+\min \left(\sqrt{n_{0}}, \sqrt{n_{1}}\right)\right) \bar{B}$.

Generalizing the above argument, all nodes in a network must fit their cycle lengths to (2) to ensure the all-pair neighbor discovery. Observe that $s_{\text {high }}$ may be way higher than the ordinary speed of nodes. For example, in the battlefields an armored vehicle can move much faster than the soldiers. The fitted cycle lengths will be dominated by $s_{\text {high }}$ and become very short. This limits the effect of power saving in practice.

\subsection{The Uni-Scheme}

Given a positive integer $z$, for each cycle length $n, n \geq z$, chosen by a station, we define a quorum $S(n, z)$, a subset of $\{0,1, \ldots, n-1\}$, as

$$
S(n, z)=\left\{0,1, \ldots,\lfloor\sqrt{n}\rfloor-1, e_{1}, \ldots, e_{p-1}\right\},
$$

where $\lfloor\sqrt{n}\rfloor-1<e_{1} \leq\lfloor\sqrt{n}\rfloor+\lfloor\sqrt{z}\rfloor-1,0<e_{i}-e_{i-1} \leq$ $\lfloor\sqrt{z}\rfloor$ for all $2 \leq i \leq p-1, p=\lfloor(n-\lfloor\sqrt{n}\rfloor) /\lfloor\sqrt{z}\rfloor\rfloor$. Basically, $S(n, z)$ contains $\lfloor\sqrt{n}\rfloor$ continuous elements starting from 0 , followed by $p-1$ interspaced elements with mutual 
distances less than or equal to $\lfloor\sqrt{z}\rfloor . S(n, z)$ is not unique with the above definition. For example, suppose $n=10$ and $z=4$, then either $\{0,1,2,4,6,8\}$ or $\{0,1,2,3,5,7,9\}$ is feasible, but $\{0,1,2,3,5,6,9\}$ is not. We call (3) the Unilateral- (Uni-) scheme. Based on (3), each node obtains its cycle pattern by following the AQPS protocol described in Section 2.

A careless glance may suggest that the Uni-scheme is very similar to the schemes proposed in studies [27], [33], [34], therefore incremental. It is true that all these schemes are based on similar combinatorics called difference sets. ${ }^{5}$ However, the changes we have made in (3) render a fundamental improvement in practice:

Theorem 3.1. Given integers $z, m$, and $n$, where $m, n \geq z$. Without the clock synchronization, two adjacent stations adopting quorums $S(m, z)$ and $S(n, z)$, respectively, are able to discover each other within a worst-case delay $l_{S(m, z), S(n, z)}=$ $(\min (m, n)+\lfloor\sqrt{z}\rfloor) \bar{B}=O(\min (m, n)) \bar{B}$.

We will formally prove this theorem in Section 4. Note $l_{S(m, z), S(n, z)}=\min \left(l_{S(m, z), S(m, z)}, l_{S(n, z), S(n, z)}\right)$. Now, consider the case shown in Fig. 4 where $H_{0}$ and $H_{1}$ need to decide their respective $n_{0}$ and $n_{1}$ such that (1) holds. Theorem 3.1 indicates that it is sufficient for any of these two nodes to reduce $l_{S(m, z), S(n, z)}$ by picking a small cycle length. The delay can be controlled unilaterally. With this observation, $H_{0}$ and $H_{1}$ can simply have their $n_{i}$ such that

$$
l_{S\left(n_{i}, z\right), S\left(n_{i}, z\right)} \leq \frac{r-d}{2 s_{i}}
$$

to ensure the in-time neighbor discovery, ${ }^{6}$ as

$$
\begin{aligned}
l_{S\left(n_{0}, z\right), S\left(n_{1}, z\right)} & =\min \left(l_{S\left(n_{0}, z\right), S\left(n_{0}, z\right)}, l_{S\left(n_{1}, z\right), S\left(n_{1}, z\right)}\right) \\
& \leq \frac{r-d}{2 \max \left(s_{0}, s_{1}\right)} \leq \frac{r-d}{s_{0}+s_{1}} .
\end{aligned}
$$

Equation (4) implies that all nodes in a network can choose the cycle lengths based on their individual speed rather than the highest possible one. Most nodes will obtain longer cycle lengths. The overall energy efficiency can be improved.

Let us look at a concrete example on the battlefield. Suppose the soldiers (i.e., nodes) have mobility ranging from $5 \mathrm{~m} / \mathrm{s}$ (when walking/running) to $30 \mathrm{~m} / \mathrm{s}$ (when carried by vehicles), $s_{\text {high }}=30 \mathrm{~m} / \mathrm{s}, r=100 \mathrm{~m}, d=60 \mathrm{~m}$, $\bar{B}=100 \mathrm{~ms}$, and $\bar{A}=25 \mathrm{~ms}$ [12]. Adopting the gridscheme, a node $H$ with speed $5 \mathrm{~m} / \mathrm{s}$ needs to fit its cycle length $n$ such that $l_{Q(n), Q(n)}=(n+\sqrt{n}) \bar{B} \leq \frac{100-60}{5+30}=1.14 \mathrm{~s}$. We have $n=4$, as only a $2 \times 2$ grid is feasible. Following the AQPS protocol, the duty cycle, $^{7}$ i.e., the minimum portion of time a station must remain awake, of $H$ is $\frac{3 \cdot \bar{B}+1 \cdot \bar{A}}{4 \bar{B}}=0.81$. On the other hand, the Uni-scheme has $z=4$ by $l_{S(z, z), S(z, z)}=(z+\lfloor\sqrt{z}\rfloor) \bar{B} \leq \frac{100-60}{2 \cdot 30}=0.67$ s. The cycle length of $H$ must satisfy $l_{S(n, 4), S(n, 4)}=(n+2) \bar{B} \leq \frac{100-60}{2 \cdot 5}=$ $4 \mathrm{~s}$. We have $n=38$, and the duty cycle of $H$ becomes 0.68 , yielding 16 percent improvement in energy efficiency.

5. We omit the discussion about difference sets due to the space limitation. Interested readers may refer to [4], [27] for more details.

6. The value of $z$ can be set by $l_{S(z, z), S(z, z)} \leq \frac{r-d}{2 s}$ to ensure that it is smaller than any picked $n$. We will study the effect of $z$ in Section 6 .

7. Readers may refer to 6LoWPAN [24], [29] for more discussions on duty cycles.
It is also interesting to note that the Uni-scheme is a generalization of the traditional grid-scheme [7], [20], [32], [35]. The Uni-scheme degenerates when $n$ is a square, $n=z$, and $e_{i}-e_{i-1}=\lfloor\sqrt{z}\rfloor$. For example, let $z=n=9$ and $e_{i}-e_{i-1}=3$, we have $S(9,9)=\{0,1,2,5,8\}$ containing a column and a row in a $3 \times 3$ grid shown in (Fig. 2). Unlike the grid-scheme, however, the Uni-scheme is defined over arbitrary values of $n$ (as long as $n \geq z$ ) rather than squares only. This provides a finer granularity for each node in selecting a proper cycle length to strike the balance between the energy efficiency and incurred neighbor discovery delay.

\section{Theoretical Foundations}

In this section, we give rigorous proof of the theorems described in Section 3 and explain their rationales. Note the definitions given in Section 4.1 are largely based on the previous arts [4], [27], [34], and are included for selfcontainment. Our contributions lay in Section 4.2.

\subsection{Definitions}

Consider the sets containing the numbers of beacon intervals. We have:

Definition 4.1 ( $n$-coterie). Given an integer $n$ and a universal set $U=\{0,1, \ldots, n-1\}$ over the modulo- $n$ plane. Let $X$ be a set of nonempty subsets of $U$. We call $X$ an $n$-coterie if and only if $\forall Q, Q^{\prime} \in X, Q \cap Q^{\prime} \neq \varnothing$.

For example, the set $\{\{0,1,2,3,6\},\{1,3,4,5,7\}\}$ given in Fig. 2 is a 9-coterie. Conventionally, a coterie $X$ is termed a quorum system, and the elements of $X$ (i.e., $Q$ ) are called the quorums.

Not every quorum system is applicable to the AQPS protocols [20]. In an AQPS protocol, two quorums must intersect even when one "shifts." This leads to the following definitions:

Definition 4.2 ( $(n, i)$-cyclic set). Given integers $n$ and $i$, $0 \leq i \leq n-1$. Let $Q$ be a subset of $U, U=\{0,1, \ldots, n-1\}$. We call $C_{n, i}(Q)$ an $(n, i)$-cyclic set of $Q$ if and only if $C_{n, i}(Q)=\{(q+i) \bmod n: \forall q \in Q\}$.

For brevity, we denote a group of cyclic sets as $C_{n}(Q)=$ $\left\{C_{n, i}(Q): \forall i\right\}$. Taking $Q=\{0,1,2,3,6\}$ as an example, we have $C_{9}(Q)=\{\{0,1,2,3,6\},\{1,2,3,4,7\}, \ldots,\{8,0,1,2,5\}\}$.

Definition 4.3 ( $n$-cyclic quorum system). Given an integer $n$ and a universal set $U=\{0,1, \ldots, n-1\}$. Let $X$ be a set of nonempty subsets of $U$. We call $X$ an $n$-cyclic quorum system if and only if $\bigcup_{Q \in X} C_{n}(Q)$ forms an $n$-coterie.

For example, the set $\{\{0,1,2,3,6\},\{1,3,4,5,7\}\}$ also forms a 9-cyclic quorum system because each pair of elements in

$$
\begin{aligned}
& \{\{0,1,2,3,6\},\{1,2,3,4,7\}, \ldots,\{8,0,1,2,5\}\} \\
& \cup\{\{1,3,4,5,7\},\{2,4,5,6,8\}, \ldots,\{0,2,3,4,6\}\}
\end{aligned}
$$

intersects. The cyclic property ensures the shift-invariant intersection. Suppose $H_{1}$ 's clock leads $H_{0}$ 's clock by 2 beacon intervals in Fig. 2 (that is, $\delta=2$ ), from $H_{0}$ 's point 


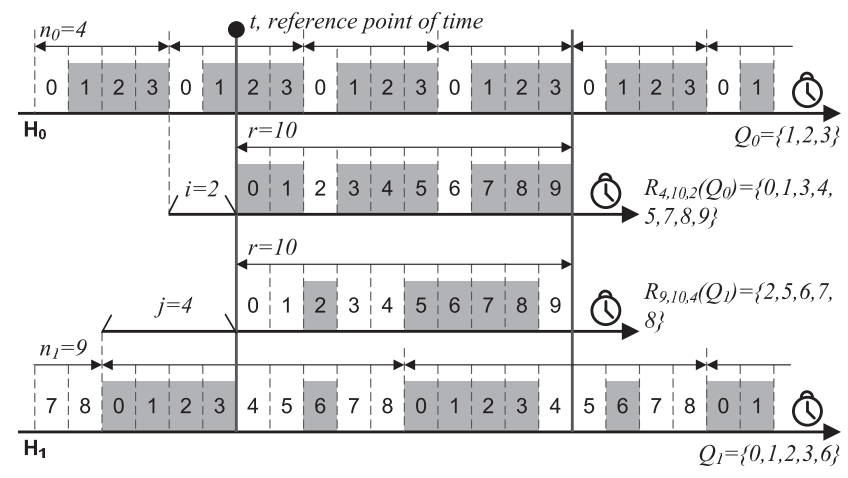

Fig. 5. The Hyper Quorum System guarantees the intersection between projections of quorums over a modulo-r plane.

of view, the quorum of $H_{1}$ becomes $C_{9,-2}(\{1,3,4,5,7\})=$ $\{8,1,2,3,5\}$ which, by definition, still belongs to $C_{9}(\{1,3$, $4,5,7\})$. We will discuss the case when $\delta \in \mathbb{R}$ later.

To allow different nodes to adopt quorums defined based on different universal sets (and therefore different power saving effect and neighbor discovery delay), the above definitions can be generalized as follows:

Definition 4.4 ( $(n, r, i)$-revolving set). Given integers $n, r$, and $i$, where $0 \leq i \leq n-1$. Let $Q$ be a subset of $U$, $U=\{0,1, \ldots, n-1\}$. We call $R_{n, r, i}(Q)$ an $(n, r, i)$-revolving set of $Q$ if and only if $R_{n, r, i}(Q)=\{(q+k n)-i: 0 \leq$ $(q+k n)-i \leq r-1, \forall q \in Q, k \in \mathbb{Z}\}$.

Intuitively, $R_{n, r, i}(Q)$ is a "projection" of $Q$ from a modulo- $n$ plane onto a modulo- $r$ plane with an index shift $i$. For example, consider a quorum $Q=\{0,1,2,3,6\}$ shown in Fig. 5, which is a subset of the universal set $U=\{0,1, \ldots, 8\}$. Given a shift index $i=4$, we may project $Q$ from the modulo-9 plane onto the modulo-10 plane by $R_{9,10,4}(Q)=$ $\{2,5,6,7,8\}$, a subset of another universal set $U^{\prime}=$ $\{0,1, \ldots, 9\}$. A revolving set $R_{n, r, i}(Q)$ degenerates into a cyclic set $C_{n,(-i \bmod n)}(Q)$ when $r=n$. For brevity, we denote a group of revolving sets as $R_{n, m}(Q)=\left\{R_{n, m, i}(Q): \forall i\right\}$.

Definition $4.5 \mathbf{(}\left(n_{0}, n_{1}, \ldots, n_{d-1} ; r\right)$-hyper quorum system (HQS)). Given integers $n_{0}, n_{1}, \ldots, n_{d-1}$ and $r$, where $d \in \mathbb{Z}$. Let $Y=\left\{Q_{0}, Q_{1}, \ldots, Q_{d-1}\right\}$ in which $Q_{i}, 0 \leq i \leq d-1$, is a nonempty subset of the universal set $U_{i}=\left\{0,1, \ldots, n_{i}-1\right\}$ over the modulo- $n_{i}$ plane. We call $Y$ an $\left(n_{0}, n_{1}, \ldots, n_{d-1} ; r\right)$ Hyper Quorum System if and only if the set $\bigcup_{Q_{i} \in Y} R_{n_{i}, r}\left(Q_{i}\right)$ forms an r-coterie.

An HQS guarantees the intersection between "projections" of quorums over a modulo plane that may be different from where the quorums reside originally. Following the example shown in Fig. 5 where $Q_{0}=\{1,2,3\}$ and $Q_{1}=\{0,1,2,5,8\}$ are adopted by $H_{0}$ and $H_{1}$, respectively. Given an arbitrary reference point of time, denoted by $t$. Suppose at $t, H_{0}$ and $H_{1}$ are in their beacon intervals 2 and 4, respectively. Then, $H_{0}$ and $H_{1}$ are guaranteed to overlap in at least one awake beacon interval within the 10 beacon intervals after $t$, since $R_{4,10,2}\left(Q_{0}\right) \cap R_{9,10,4}\left(Q_{1}\right) \neq \varnothing$. Actually, we may easily verify that given any reference point of time, where $H_{0}$ and $H_{1}$ are in their beacon intervals $i$ and $j$, respectively, $H_{0}$ and $H_{1}$ are guaranteed to overlap within 10 beacon intervals. Therefore, the set $\{\{1,2,3\},\{0,1,2,5,8\}\}$ is a $(4,9 ; 10)$-HQS. Nodes with an HQS can obtain cycle patterns of different lengths without losing the network connectivity.

\subsection{Proof of Theorem 3.1}

Define the heads of a revolving set $R_{n, r, i}(Q)$ as those elements projected from the smallest element in $Q$. For example, in Fig. 5 the elements 3 and 7 are heads of $R_{4,10,2}(\{1,2,3\})$. There could be none, or more than one head.

Lemma 4.6. Given positive integers $m, n$, and $z$, where $m, n \geq z$. The set $\{S(m, z), S(n, z)\}$ based on (3) forms an $(m, n ; \min (m, n)+\lfloor\sqrt{z}\rfloor-1)$-hyper quorum system.

Proof. Without loss of generality, let $m \leq n$, and $r=m+$ $\lfloor\sqrt{z}\rfloor-1$. We show that $\forall i, j, 0 \leq i \leq m$ and $0 \leq j \leq n$, $R_{m, r, i}(S(m, z)) \cap R_{n, r, j}(S(n, z)) \neq \varnothing$. Denote $h$ the first head in $R_{m, r, i}(S(m, z))$. Since $r \geq m, h$ exists and $h \leq m-1$. If $h$ is included in $R_{n, r, j}(S(n, z))$, we finish the proof. Otherwise, consider two elements $s$ and $t$ in $R_{n, r, j}(S(n, z))$ such that $s<h<t$. By definition of $S(n, z)$, any two interspaced elements in $R_{n, r, j}(S(n, z))$ must have mutual distance less than or equal to $\lfloor\sqrt{z}\rfloor$. We have $t \leq s+\lfloor\sqrt{z}\rfloor$, leading to $t \leq h+\lfloor\sqrt{z}\rfloor-1 \leq(m-1)+$ $\lfloor\sqrt{z}\rfloor-1=r-1$. The element $t$ exists. On the other hand, by definition of $S(m, z), h$ is a head of $R_{m, r, i}(S(m, z))$ implies that there exist $\lfloor\sqrt{m}\rfloor-1$ continuous elements after $h$ in $R_{m, r, i}(S(m, z))$. Since $t \leq h+$ $\lfloor\sqrt{z}\rfloor-1 \leq h+\lfloor\sqrt{m}\rfloor-1$, the element $t$ must also be included in $R_{m, r, i}(S(m, z))$. We have $R_{m, r, i}(S(m, z)) \cap$ $R_{n, r, j}(S(n, z)) \neq \varnothing$.

The above lemma indicates that under the situation where there is no clock shift between stations or the clock shifts are multiples of a $\bar{B}$, two adjacent nodes adopting quorums $S(m, z)$ and $S(n, z)$, respectively, are guaranteed to discover each other within $\min (m, n)+\lfloor\sqrt{z}\rfloor-1$ beacon intervals. Study [20] has further pointed out that:

Lemma 4.7. Two stations having an arbitrary clock shift $\delta \bar{B}$, $\delta \in \mathbb{R}$, and remaining awake/sleep based on the AQPS protocol described in Section 2 are guaranteed to discover each other within $l \bar{B}$ if given any clock shift $i \bar{B}, i \in \mathbb{Z}$, the two stations can discover each other within $(l-1) \bar{B}$.

Theorem 3.1 is a direct consequence of Lemmas 4.6 and 4.7.

\section{Unilateral Wakeup for Group Mobility}

Group mobility is one of the prominent characteristics of MANETs and is a main factor affecting link discovery, route establishment, and clustering [3], [6], [17]. In this section, we show that the Uni-Scheme can be extended to improve the energy efficiency of MANETs further, if group mobility is present. Our idea, based on a common observation that nodes moving in the same group usually have slow relative speed independent of their absolute speed [3], [17], [30], is to allow nodes in a group to obtain the cycle length corresponding to the relative speed of nodes within that group, rather than their individual absolute speed, to save energy. 


\subsection{The Extended Uni-Scheme}

Currently, it is problematic to apply a wakeup scheme to MANETs with group mobility. These networks are commonly divided into clusters [3], [15], [26], [28], [30], as shown in Fig. 3b, where each cluster corresponds to a moving group. Studies [25], [33], [35] have proposed wakeup schemes for clustered networks that differentiate the quorums between members and clusterheads/relays, and allow the members to sleep more while being able to discover their clusterheads without losing the network connectivity. Specifically, given an integer $n$, the study [33] defines a new quorum for members, a subset of $\{0,1, \ldots, n-1\}$, as

$$
A(n)=\left\{e_{0}, e_{1}, \ldots, e_{p-1}\right\}
$$

where $e_{0}=0,0<e_{i}-e_{i-1} \leq\lfloor\sqrt{n}\rfloor$ for all $1 \leq i \leq p-1$, and $p=\lceil n /\lfloor\sqrt{n}\rfloor\rceil$. The merit of $A(n)$ is that its size is less than half of that of the quorums on clusterheads/relays. However, as we have seen in Section 3.1, the clusterheads/relays (which adopt traditional quorums) can only pick very short cycle lengths due to (2). Recall that all members in each cluster must pick the same cycle length as their clusterhead does. The power saving effect is severely limited in the MANETs with group mobility. It remains challenging to design a wakeup scheme that saves energy in presence of group mobility.

In this paper, we carefully design $S(n, z)$ such that it can work with $A(n)$ to allow members to adopt $A(n)$ but avoid the limitation of short cycle lengths. ${ }^{8}$ Specifically, a network starts from a flat topology where each node adopts a quorum $S(n, z)$ defined by (3) based on its own speed. After clusters are formed at either the network [3], [15], [26], [28] or application [30] layer, a member can be informed of the cycle length $n$ decided by its clusterhead and adopt $A(n)$. We show that:

Theorem 5.1. Given integers $z$ and $n$, where $n \geq z$. Without the clock synchronization, two adjacent stations adopting quorums $S(n, z)$ and $A(n)$, respectively, are able to discover each other within a worst-case delay $l_{S(n, z), A(n)}=(n+1) \bar{B}=O(n) \bar{B}$.

There is no guarantee for the neighbor discovery between members. This is fine as long as each clusterhead can discover its members and nearby relays [33], [35]. The clusterhead can forward the existence of members to allow intracluster communication; while sending/receiving data to/from relays to allow intercluster communication. We can see that:

1. To ensure that all relays in a network can be discovered by a clusterhead in-time, each relay, by Theorem 3.1, can pick a conservative cycle length satisfying (2) unilaterally regardless of the one decided by the clusterhead;

2. To ensure that the members of a cluster can be discovered by the clusterhead in-time, by Theorem 5.1 it is sufficient for the clusterhead and members to adopt a cycle length $n$ such that

8. Actually, $S(n, z)$ can work with other existing quorums dedicated for members [25], [35]. Here, our focus is $S(n, z)$ hence further discussions are omitted.

$$
l_{S(n, z), A(n)} \leq \frac{r-d}{s_{r e l}},
$$

where $s_{\text {rel }}$ is the highest relative speed between the clusterhead and members.

Studies [3], [17], [30] show that nodes moving in the same group usually have slow relative speed independent of their absolute speed. The cycle lengths determined by the clusterhead and members can be consistently long regardless of the mobility of their cluster. Since members are the majority of nodes in a network, the overall saving in energy consumption can be improved.

Following the previous battlefield example where $s_{\text {high }}=$ $30 \mathrm{~m} / \mathrm{s}, r=100 \mathrm{~m}, d=60 \mathrm{~m}, \bar{B}=100 \mathrm{~ms}$, and $\bar{A}=25 \mathrm{~ms}$. It is common that the soldiers move in groups (e.g., when marching or carried by vehicles). Suppose the relative speed of nodes within a group is no above than $4 \mathrm{~m} / \mathrm{s}$. In traditional grid-scheme, the cycle lengths $m$ and $n$ picked by a relay and clusterhead moving in speed $5 \mathrm{~m} / \mathrm{s}$, respectively, must both satisfy $l_{Q(n), Q(n)} \leq \frac{100-60}{5+30}=1.14 \mathrm{~s}$. Only the $2 \times 2$ grid is feasible and we have $m=n=4$. By definition, members of the clusterhead must also pick $n=4$ to form their quorums. The duty cycles of the clusterhead/relay and members are 0.81 and $\frac{2 \cdot \bar{B}+2 \cdot \bar{A}}{4 \cdot \bar{B}}=0.63$, respectively. On the other hand, the Uni-scheme (having $z=4$ ) allows the relay to adopt $n=9$ to satisfy $l_{S(n, 4), S(n, 4)} \leq \frac{100-60}{5+30}=1.14 \mathrm{~s}$, and the clusterhead to pick $n=99$ by $l_{S(n, 4), A(n)}=(n+$ 1) $\bar{B} \leq \frac{100-60}{4}=10 \mathrm{~s}$. The members also have $n=99$. In this case, the duty cycles of the relay, clusterhead, and members become $0.75,0.66$, and 0.34 , respectively, yielding 7, 19, and 46 percent improvement in energy efficiency.

\subsection{Proof of Theorem $\mathbf{5 . 1}$}

Theorem 5.1 can be proofed by either the difference sets [27] or cyclic bicoteries [4]. Here, we present the latter to stay consistent with our previous discussions.

Definition 5.2 ( $n$-cyclic bicoterie). Given integers $n$ and $a$ universal set $U=0,1, \ldots, n-1$. Let $X$ and $Y$ be sets of nonempty subsets of $U$. We call the pair $(X, Y)$ an $n$-cyclic bicoterie if and only if $\forall i, j, Q \in X, Q^{\prime} \in Y, C_{n, i}(Q) \cap$ $C_{n, j}\left(Q^{\prime}\right) \neq \varnothing$.

Lemma 5.3. Given positive integers $n$, and $z$, where $n \geq z$. The set $\{S(n, z), A(n)\}$ based on (3) and (5) forms an $n$-cyclic bicoterie.

Proof. We show that $\forall i, j, C_{n, i}(S(n, z)) \bigcap C_{n, j}(A(n)) \neq \varnothing$ using two steps: Step 1). Let $r=n+\lfloor\sqrt{z}\rfloor-1$. Denote $h$ the first head in $R_{n, r,-i}(S(n, z))$. Since $r \geq n, h$ exists and $h \leq n-1$. If $h$ is included in $R_{n, r,-j}(A(n))$, we go to step 2. Otherwise, consider two elements $s$ and $t$ in $R_{n, r,-j}(A(n))$ such that $s<h<t$. By definition of $A(n)$, any two interspaced elements in $R_{n, r,-j}(A(n))$ must have mutual distance less than or equal to $\lfloor\sqrt{z}\rfloor$. We have $t \leq s+\lfloor\sqrt{z}\rfloor$, leading to $t \leq h+\lfloor\sqrt{z}\rfloor-1 \leq(n-$ $1)+\lfloor\sqrt{z}\rfloor-1=r-1$. The element $t$ exists. On the other hand, by definition of $S(n, z), h$ is a head of $R_{n, r,-i}(S(n, z))$ implies that there exist $\lfloor\sqrt{n}\rfloor-1$ continuous elements after $h$ in $R_{n, r,-i}(S(n, z))$. Since $t \leq$ $h+\lfloor\sqrt{z}\rfloor-1 \leq h+\lfloor\sqrt{n}\rfloor-1$, the element $t$ must also be included in $R_{n, r,-i}(S(n, z))$. Step 2). Now, we have $R_{n, r,-i}(S(n, z)) \cap R_{n, r,-j}(A(n)) \neq \varnothing$. Let $w, 0 \leq w \leq r-1$, 


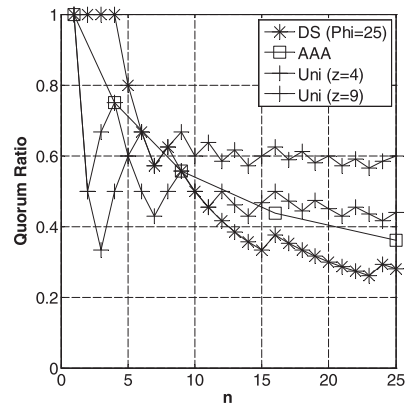

(a)

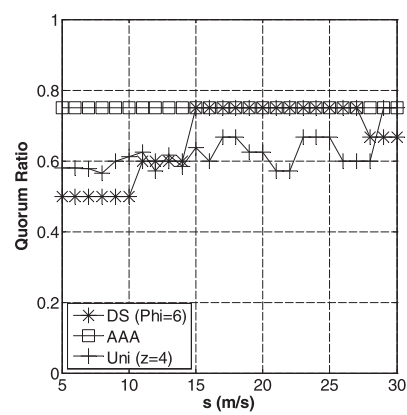

(c)

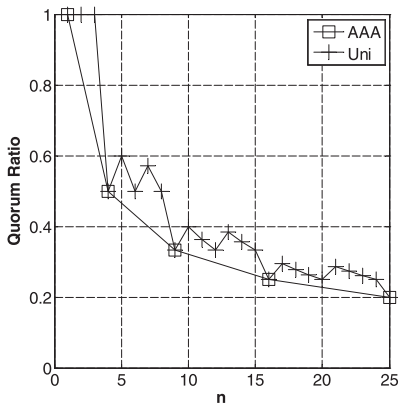

(b)

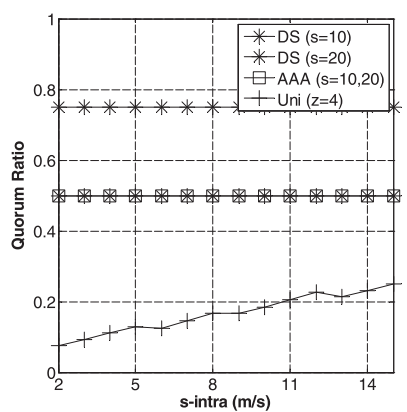

(d)

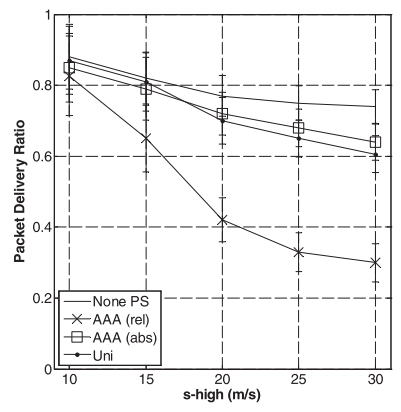

(a)

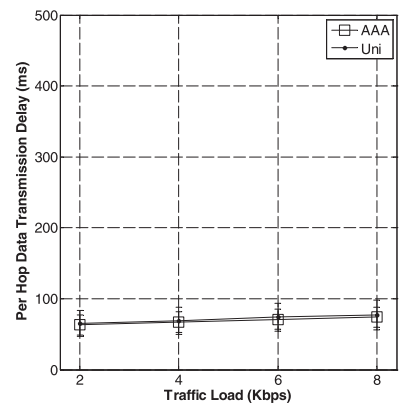

(c)

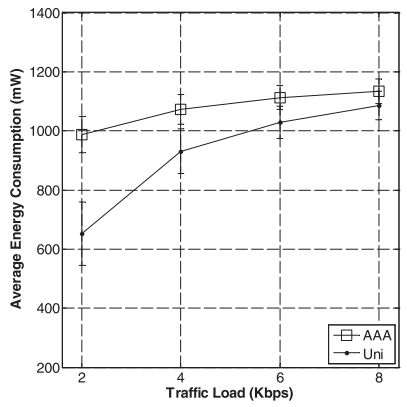

(e)

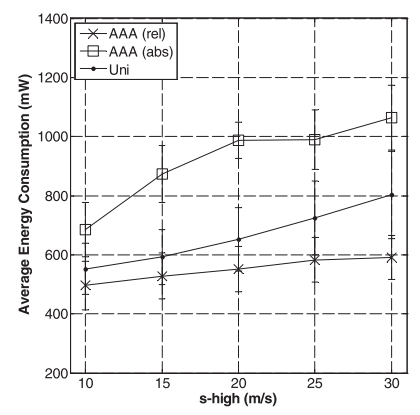

(b)

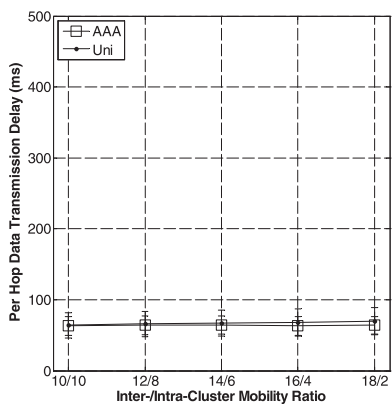

(d)

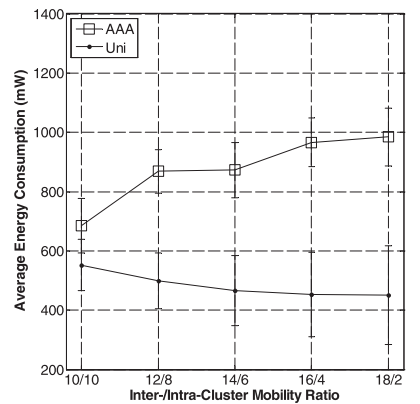

(f) (respectively, $\left.C_{n, j}(A(n))\right)$ in the tail. If $w \leq n-1$, we finish the proof. Otherwise, for any $w, n \leq w \leq r-1$, there must exist a corresponding element $w-n$ in $R_{n, r, 0}\left(C_{n, i}(S(n, z))\right)$ (respectively, $R_{n, r, 0}\left(C_{n, j}(A(n))\right)$ ). The element $w-n$ is a common element of $C_{n, i}(S(n, z))$ and $C_{n, j}(A(n))$, implying $C_{n, i}(S(n, z)) \cap C_{n, j}(A(n)) \neq \varnothing$.

The above lemma indicates that under the situation where there is no clock shift between stations or the clock shifts are multiples of a $\bar{B}$, two adjacent nodes adopting quorums $S(n, z)$ and $A(n)$, respectively, are guaranteed to discover each other within $n$ beacon intervals. Combining Lemma 4.7, we obtain the proof.

\section{Performance Evaluation}

In this section, we evaluate the performance of Uni-scheme by both the theoretical analysis and simulation. The results of analysis are presented in Fig. 6 and explained in Section 6.1, while the simulation results are shown in Fig. 7 and elaborated in Sections 6.2 and 6.3.

We implement the simulation using the $n s-2$ simulator [1] with CMU wireless extension. The simulation is conducted in a $1,000 \times 1,000 \mathrm{~m}^{2}$ field with 50 randomly distributed nodes. Each node has half-duplex wireless
Fig. 7. Simulation results. Effects of $s_{\text {high }}$ on (a) data packet delivery ratio and (b) average energy consumption. Effects of traffic load on (c) data transmission delay and (e) energy consumption. Effects of $s_{\text {high }} / s_{\text {intra }}$ on (d) data transmission delay and (f) energy consumption.

channel of rate $2 \mathrm{Mbps}$ and transmission range $100 \mathrm{~m}$. We set the radius of discovery zone $60 \mathrm{~m}$. The beacon intervals and ATIM windows are set 100 and $25 \mathrm{~ms}$, respectively. The power consumption rates of the wireless module are set $1,650,1,400,1,150$, and $45 \mathrm{~mW}$ in transmit, receive, idle, and sleep modes, respectively [22]. There are 20 sources sending packets to 20 receivers at constant bit rate ranging from 2 to 8 Kbps. Packets have size 256 bytes and are routed by the Dynamic Source Routing (DSR) protocol [21]. We do not synchronize the clocks between nodes.

To implement the node mobility, we employ the Reference Point Group Mobility (RPGM) model [17] as it covers many other popular models including the Random Waypoint, Column, Nomadic, and Pursue models [6]. Nodes are divided evenly into five groups. In each group, the reference points of nodes are randomly distributed within $50 \mathrm{~m}$ around the center, and each node moves within $50 \mathrm{~m}$ around its own reference point by following the random waypoint mobility model with speed uniformly 
distributed between $\left(0, s_{\text {intra }}\right]$. Groups in the network move by following the random waypoint model with speed uniformly distributed between $\left(0, s_{\text {high }}\right]$. We adopt MOBIC [3] as the clustering scheme since it is effective in localizing the node dynamics. Note with the above settings, nodes in the same group may have distance $(200 \mathrm{~m}$ at most) longer than their coverage. Multiple clusters can be formed in a moving group.

\subsection{Theoretical Analysis: the Quorum Ratio}

We first compare the Uni-scheme with the others that allow nodes to pick different cycle lengths to control the neighbor discovery delay, including the DS- [34] and AAA-schemes [35]. The AAA-scheme constructs a quorum system using the $\sqrt{n} \times \sqrt{n}$ grids as we have seen in Section 2.2. One advantage of the AAA-scheme is that it generates different types of quorums for the clusterheads/relays and members, respectively, thus can be applied to MANETs that are clustered based on the group mobility. Note that the AAAscheme is a generalization of various grid-/torus-schemes [7], [20], [32] so we effectively compare the Uni-scheme with all these studies. The DS-scheme constructs a quorum system based on the difference set. As compared to the AAA-scheme that requires the cycle length $n$ picked by each node to be a square, the DS-scheme allows each node to choose an arbitrary $n$, thereby offering finer granularity to the tradeoff between energy conservation and delay. However, the DS-scheme assumes a flat network and does not differentiate quorums in presence of group mobility.

To see the power saving effect given purely by a wakeup scheme without the involvement of protocol design (which we will see later), we define a theoretical metrics, the quorum ratio, that denote the proportion of beacon intervals in a cycle where a station is required to awake. Specifically, it is defined as $|Q| / n$, where $|Q|$ is the quorum size and $n$ is the cycle length. The smaller the quorum ratio, the more the power saving achievable by an AQPS protocol.

Quorum ratios over cycle lengths. Figs. $6 \mathrm{a}$ and $6 \mathrm{~b}$ show the quorum ratios given by the schemes taking different cycle lengths as the input. Basically, the longer the cycle length, the lower the quorum ratio. The nodes in a flat network or the clusterheads/relays in a clustered environment that adopt the quorums ensuring all-pair neighbor discovery can obtain the ratios shown in Fig. 6a. The DS-scheme is able to yield the lowest quorum ratios given a cycle length picked on a station. On the other hand, the AAA- and Unischeme allow members in a clustered network to obtain the improved quorum ratios shown in Fig. $6 \mathrm{~b}$ by releasing the overlap guarantee between members themselves. The quorum ratios given by AAA and Uni are lower than those of DS when the network topology is asymmetric.

Quorum ratios over delay. It is important to note that in MANETs, a scheme giving smaller quorum ratios over cycle lengths does not necessarily result in better energy efficiency. Given the cycle lengths $m$ and $n$ on two stations, the DS-, AAA-, and Uni-schemes result in the worst-case neighbor discovery delay $(\max (m, n)+\lfloor(\min (m, n)-1) / 2\rfloor+\phi) \bar{B}$, $(\max (m, n)+\min (\sqrt{m}, \sqrt{n})) \bar{B}$, and $(\min (m, n)+\lfloor\sqrt{z}\rfloor) \bar{B}$, respectively, where $\phi$ and $z$ are constants. Figs. 6c and 6d exhibit the lowest quorum ratios given by these schemes that satisfy the delay requirements under different node speed.
The ratios on stations in a flat network or the clusterheads/ relays in a clustered environment are shown in Fig. 6c, where $s$ denotes the absolute moving speed. Due to the assumption that $n$ is a square, in AAA only the $2 \times 2$ grid is feasible to (2) for all $s$, and the quorum ratios remain 0.75 . By taking arbitrary cycle lengths, both the DS- and Unischeme fit $n$ to a particular $s$ with better granularity, thereby improving the ratios. Notice that the DS-scheme does not outperform Uni with its superior quorum ratios over cycle lengths (as shown in Fig. 6a). This is because that the Unischeme shortens the neighbor discovery delay from $O(\max (m, n))$ to $O(\min (m, n))$, so given any $s$ it is able to fit a longer cycle length using (4) rather than (2) as DS does. In Fig. 6c, the values of $n$ given by the Uni-scheme range from 4 (when $s=30$ ) to 38 (when $s=5$ ), which are much wider than those (ranging from 4 to 6 ) given by DS. This also avoids a common drawback of most schemes that the quorum ratio fluctuates sharply when $n$ is small (especially when $n<10$ ). The Uni-scheme render more stable quorum ratios than DS, and consistently improves the ratios of AAA (up to 24 percent) across all speed.

Quorum ratios over group mobility. Fig. 6d shows the lowest quorum ratios given by different schemes for members in a clustered network. We vary the relative speed $s_{\text {intra }}$ of nodes within a cluster from 2 to $15 \mathrm{~m} / \mathrm{s}$ while keeping their absolute speed $s=10$ and $20 \mathrm{~m} / \mathrm{s}$, respectively. Since the delays in DS- and AAA-schemes cannot be controlled unilaterally, the relays and clusterhead (so do its members) must pick a cycle length subject to $s$ rather than $s_{\text {intra }}$ using (2). Therefore, the quorum ratios on members remain the same for all $s_{\text {intra }}$. On the other hand, the delays in Uni-scheme can be unilaterally controlled by relays, so the clusterhead and members can pick a cycle length corresponding to $s_{i n t r a}$ using (6). The quorum ratios are lowered as $s_{\text {intra }}$ decreases (up to 89 and 84 percent against DS and AAA, respectively), and these lowered ratios are independent with $s$.

From the above, we can see that in either the flat or clustered networks, the Uni-scheme allows nodes with slower moving speed to save more energy.

\subsection{Energy Conservation and Data Delivery}

Next, we examine the simulation results and compare the Uni-scheme with AAA [35]. Currently, these are the only two schemes whose AQPS protocols satisfy the asynchronous, adaptive, and asymmetric requirements of MANETs [35]. Each simulation point is averaged from 10 simulation runs of duration 1,800 seconds. We also report 95 percent confidence intervals $[\mu-2.26 \mathrm{~s} / \sqrt{10}, \mu-2.26 \mathrm{~s} / \sqrt{10}]$ to the simulation points by following the Student's $t$-distribution with 9 degrees of freedom, where $\mu$ and $s^{2}$ are sample mean and variance, respectively.

Energy conservation. We first evaluate the energy efficiency under different mobility levels. We vary $s_{\text {high }}$ from 10 to $30 \mathrm{~m} / \mathrm{s}$ and keep $s_{\text {intra }}=10 \mathrm{~m} / \mathrm{s}$. Note when $s_{\text {high }}=s_{\text {intra }}$, the delay requirements for relays and clusterheads/members are identical, and the power saving effect given by a scheme is similar to those given in a flat network. Due to the space limitation, we do not show the results for a flat network. In AAA, each node adjusts its own cycle length dynamically based on two strategies. One (denoted as 
AAA(abs)) follows (2) on all nodes, and another (denoted as AAA(rel)) follows (2) on the relays and (6) on the rest. In Uni, nodes adjust $n$ using the same strategy as in AAA(rel).

As we can see from Fig. 7b, AAA(rel) and Uni save considerably more energy than AAA(abs). This is because that in $\mathrm{AAA}(\mathrm{rel})$ and Uni, only the relays are required to pick small cycle lengths when $s_{\text {high }}$ increases. Other nodes (especially members) can still save energy by taking advantages of a slow $s_{\text {intra }}$.

Data delivery. However, as shown in Fig. 7a, AAA(rel) results in poor data delivery ratio because it is not sufficient to guarantee in-time neighbor discovery between clusters by only shrinking the cycle lengths of relays. To allow communication between clusters, the clusterheads must also pick small cycle lengths, as shown by AAA(abs). This casts the energy consumption overhead to all member nodes. Since members are the majority of nodes in a network, the overall extra energy consumption can be significant. The Uni-scheme avoids this problem and is able to give 34 percent improvement in energy efficiency as compared with AAA(abs) when $s_{\text {high }}=20 \mathrm{~m} / \mathrm{s}$ while guaranteeing the data delivery.

\subsection{Effects of Traffic Loads and Group Mobility}

In this section, we investigate the effects of traffic loads and group mobility on AQPS protocols using the AAA and Uni schemes, respectively. We focus on the AAA(abs) here.

Data transmission delay. The end-to-end data transmission delays incurred by AAA and Uni are similar, which both range from 0.4 to 2 seconds in general cases. The high jitter is resulted largely from the routing protocol at Network layer rather than the AQPS protocols at MAC layer. To see this, we study the per hop data transmission delays caused at the MAC layer under different traffic loads and group mobility. The results are shown in Figs. 7c and 7d. In most cases, the average per hop delay is below $100 \mathrm{~ms}$, with a slight increase at higher traffic loads due to the contention, and is invariant under different mobility levels. This is because that an AQPS protocol (either AAA- or Uni-based) requires each node to remain awake during the ATIM window in every beacon interval. So after discovery, a sender only needs to buffer the data until the coming of the next ATIM window at the receiver side. The data buffering delay can be no longer than a beacon interval (100 ms).

Energy conservation. As shown in Fig. 7e, the energy consumption rate increases in both AAA and Uni as the traffic load becomes higher. This is due to the frequent ATIM notification and data transmission procedures. As most existing wakeup schemes, the power saving effect given by the Uni-scheme takes place only at idle time without sacrificing data transmissions.

However, different from existing schemes, the Unischeme renders opposite energy saving tendency as the group mobility becomes prominent. By increasing the ratio $s_{\text {high }} / s_{\text {intra }}$ as shown in Fig. $7 \mathrm{f}$, the Uni-scheme is able to yield more energy saving opposing to AAA where the energy efficiency decreases. This is because that in AAA all nodes need to adopt smaller cycle lengths as $s_{\text {high }}$ grows; but in Uni the members can adopt longer cycle lengths according to $s_{\text {intra }}$ regardless of $s_{\text {high }}$. Again, since the members are the majority of nodes, the overall energy consumption rate can be reduced. In particular, at an extreme case where $s_{\text {high }}=18$ and $s_{\text {intra }}=2 \mathrm{~m} / \mathrm{s}$, the Uni-scheme is able to yield 54 percent improvement in energy efficiency as compared with AAA.

\section{Conclusions}

In this paper, we investigated the impact of node mobility to the asynchronous wakeup protocols. We identified several shortcomings of existing quorum schemes if to be applied to the MANETs, and proposed the Uni-scheme. The Uni-scheme contributes in the following aspects:

- The concept of unilateral wakeup is introduced that allows nodes with slower moving speed to save more energy. The Uni-scheme shortens the neighbor discovery delay from $O(\max (m, n))$ to $O(\min (m, n))$ so each node can select the cycle length based on its own speed rather than the highest possible one in the network.

- The Uni-scheme is applicable to MANETs with group mobility. The Uni-scheme is carefully designed such that it is compatible with existing quorum schemes for clustered networks. The Unischeme allows nodes in a moving group to pick cycle lengths based on the relative speed in the group (which is usually slow) rather than the relative speed between groups.

- Simulation results show that the Uni-scheme is able to render significant improvement in energy efficiency while guaranteeing the network connectivity.

We plan to implement the Uni-scheme-based wakeup protocols on real sensors in the future.

\section{References}

[1] "The Network Simulator - ns-2," http://www.isi.edu/nsnam/ns, 2013.

[2] J. Aslam, Z. Butler, F. Constantin, V. Crespi, G. Cybenko, and D. Rus, "Tracking a Moving Object with a Binary Sensor Network," Proc. First Int'l Conf. Embedded Networked Sensor Systems (SenSys), pp. 150-161, 2003.

[3] P. Basu, N. Khan, and T. Little, "A Mobility Based Metric for Clustering in Mobile Ad Hoc Networks," Proc. Int'l Distributed Systems Workshop (ICDCSW), pp. 413-418, 2001.

[4] P. Bernstein, V. Hadzilacos, and N. Goodman, Concurrency Control and Recovery in Database Systems. Addison-Wesley, 1987.

[5] J. Blum, A. Eskandarian, and L. Hoffman, "Challenges of Intervehicle Ad Hoc Networks," IEEE Trans. Intelligent Transportation Systems, vol. 5, no. 4, pp. 347-351, Dec. 2004.

[6] T. Camp, J. Boleng, and V. Davies, "A Survey of Mobility Models for Ad Hoc Network Research," Wireless Comm. Mobile Computing, vol. 2, no. 5, pp. 483-502, 2002.

[7] C. Chao, J. Sheu, and I. Chou, "An Adaptive Quorum-Based Energy Conserving Protocol for IEEE 802.11 Ad Hoc Netowrks," IEEE Trans. Mobile Computing, vol. 5, no. 5, pp. 560-570, May 2006.

[8] W. Chen, N. Jain, and S. Singh, "ANMP: Ad Hoc Network Management Protocol," IEEE J. Selected Areas Comm., vol. 17, no. 8, pp. 1506-1531, Aug. 1999.

[9] B. Choi and X. Shen, "Adaptive Asynchronous Sleep Scheduling Protocols for Delay Tolerant Networks," IEEE Trans. Mobile Computing, vol. 10, no. 9, pp. 1283-1296, Sept. 2011.

[10] B. Choi and X. Shen, "Adaptive Asynchronous Clock Based Power Saving Protocols for Delay Tolerant Networks," Proc. IEEE Global Comm Conf. (GlobeCom), 2010.

[11] Z. Chou, "Optimal Adaptive Power Management Protocols for Asynchronous Wireless Ad Hoc Networks," Proc. IEEE Wireless Comm. Networking Conf. (WCNC), pp. 61-65, 2007.

[12] IEEE Standard 802.11-1999, Part 11: Wireless LAN Medium Access Control (MAC) and Physical Layer (PHY) Specifications, IEEE, 1999. 
[13] N. Din, G. Tan, H. Ma, M. Lin, and Y. Shang, "Low-Power Vehicle Speed Estimation Algorithm Based on WSN," Proc. IEEE Int'l Conf. Intelligent Transport Systems (ITSC), pp. 1015-1020, 2008.

[14] J. Ding, S. Cheung, C. Tan, and P. Varaiya, "Signal Processing of Sensor Node Data for Vehicle Detection," Proc. IEEE Seventh Int'l Conf. Intelligent Transport Systems (ITSC), pp. 70-75, 2004.

[15] I. Er and W. Seah, "Mobility-Based D-Hop Clustering Algorithm for Mobile Ad Hoc Networks," Proc. IEEE Wireless Comm. Networking Conf. (WCNC), pp. 2359-2364, 2004.

[16] L. Feeney and M. Nilsson, "Investigating the Energy Consumption of a Wireless Network Interface in an Ad Hoc Networking Environment," Proc. IEEE INFOCOM, pp. 1548-1557, 2001.

[17] X. Hong, M. Gerla, G. Pei, and C. Chiang, "A Group Mobility Model for Ad Hoc Wireless Networks," Proc. Second ACM Int'l Workshop Modeling, Analysis and Simulation Wireless and Mobile Systems (MSWiM), pp. 53-60, 1999.

[18] T. Hou and T. Tsai, "An Access-Based Clustering Protocol for Multihop Wireless Ad Hoc Networks," IEEE J. Selected Areas in Comm., vol. 19, no. 7, pp. 1201-1210, July 2001.

[19] A. Iwata, C. Chiang, G. Pei, M. Gerla, and T. Chen, "Scalable Routing Strategies for Ad Hoc Wireless Networks," IEEE J. Selected Areas Comm., vol. 17, no. 8, pp. 1369-1379, Aug. 1999.

[20] J. Jiang, Y. Tseng, C. Hsu, and T. Lai, "Quorum-Based Asynchronous Power-Saving Protocols for IEEE 802.11 Ad Hoc Networks," Mobile Networks and Applications, vol. 10, nos. 1/2, pp. 169-181, 2005.

[21] D. Johnson and D. Maltz, "Dynamic Source Routing in Ad Hoc Wireless Networks," Mobile Computing, vol. 353, pp. 153-181, 1996.

[22] E. Jung and N. Vaidya, "An Energy Efficient Mac Protocol for Wireless Lans," Proc. IEEE INFOCOM, pp. 1756-1764, 2002.

[23] R. Kravets and P. Krishnan, "Application-Driven Power Management for Mobile Communication," Wireless Networks, vol. 6, no. 4, pp. 263-277, 2000.

[24] N. Kushalnagar, C. Schumacher, and G. Montenegro, IPv6 over Low-Power Wireless Personal Area Networks (6LoWPANs): Overview, Assumptions, Problem Statement, and Goals, IETF RFC 4919, 2007.

[25] S. Lai, B. Ravindran, and H. Cho, "Heterogenous Quorum-Based Wake-Up Scheduling in Wireless Sensor Networks," IEEE Trans. Computer, vol. 59, no. 11, pp. 1562-1575, Nov. 2010.

[26] C. Lin and M. Gerla, "Adaptive Clustering for Mobile Wireless Networks," IEEE J. Selected Areas Comm., vol. 15, no. 7, pp. 12651275, Sept. 1997.

[27] W. Luk and T. Wong, "Two New Quorum Based Algorithms for Distributed Mutual Exclusion," Proc. 17th Int'l Conf. Distributed Systems (ICDCS), pp. 100-106, 1997.

[28] A. McDonald and T. Znati, "A Mobility-Based Framework for Adaptive Clustering in Wireless Ad Hoc Networks," IEEE J. Selected Areas Comm., vol. 17, no. 8, pp. 1466-1487, Aug. 1999.

[29] G. Montenegro, N. Kushalnagar, J. Hui, and D. Culler, Transmission of IPv6 Packets over IEEE 802.15.4 Networks, IETF RFC 4944, 2007.

[30] H. Reumerman, M. Roggero, and M. Ruffini, "The ApplicationBased Clustering Concept and Requirements for Intervehicle Networks," IEEE Comm. Magazine, vol. 43, no. 4, pp. 108-113, Apr. 2005.

[31] P. Sinha, R. Sivakumar, and V. Bharghavan, "Enhancing Ad Hoc Routing with Dynamic Virtual Infrastructures," Proc. IEEE INFOCOM, pp. 1763-1772, 2001.

[32] Y. Tseng, C. Hsu, and T. Hsieh, "Power-Saving Protocols for IEEE 802.11-Based Multi-Hop Ad Hoc Networks," Proc. IEEE INFOCOM, pp. 200-209, 2002.

[33] S. Wu, C. Chen, and M. Chen, "An Asymmetric Quorum-Based Power Saving Protocol for Clustered Ad Hoc Networks," Proc. 27th Int'l Conf. Distributed Systems (ICDCS), pp. 1-8, 2007.

[34] S. Wu, C. Chen, and M. Chen, "Fully Adaptive Power Saving Protocols for Ad Hoc Networks Using the Hyper Quorum System," Proc. Int'l Conf. Distributed Systems (ICDCS), pp. 785-792, 2008.

[35] S. Wu, C. Chen, and M. Chen, "AAA: Asynchronous, Adaptive, and Asymmetric Power Management for Mobile Ad Hoc Networks," Proc. IEEE INFOCOM, pp. 2541-2545, 2009.

[36] S. Wu, J. Sheu, and C. King, "Unilateral Wakeup for Mobile Ad Hoc Networks," Proc. Int'l Conf. Parallel Processing (ICPP), pp. 130137, 2011.

[37] R. Zheng, J. Hou, and L. Sha, “Optimal Block Design for Asynchronous Wake-Up Schedules and Its Applications in Multihop Wireless Networks," IEEE Trans. Mobile Computing, vol. 5, no. 9, pp. 1228-1241, Sept. 2006.

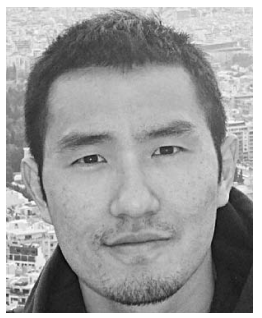

Shan-Hung Wu received the BS degree from the Department of Information Management, National Central University, Jhongli, Taiwan, the MS degree from the Department of Computer Science and Information, National Taiwan University, Taipei, Taiwan, and the PhD degree from the Department of Electrical Engineering, National Taiwan University, Taipei, Taiwan. He is currently an assistant professor in the Department of Computer Science, National Tsing Hua University, Hsinchu, Taiwan. Before joining National Tsing Hua University, he was a senior research scientist at Telcordia Technologies and led several Telcordia projects including the clustered in-memory database, video surveillance workbench, and the rating engine of Telcordia ISCP platform. His research interests include wireless networks, database systems, data mining, and mobile data management.

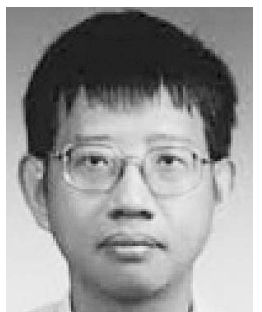

Jang-Ping Sheu received the BS degree in computer science from Tamkang University, Taiwan, Republic of China, in 1981, and the $\mathrm{MS}$ and $\mathrm{PhD}$ degrees in computer science from National Tsing Hua University, Taiwan, Republic of China, in 1983 and 1987, respectively. He is currently a chair professor in the Department of Computer Science, National Tsing Hua University. He was a chair of the Department of Computer Science and Information Engineering, National Central University, from 1997 to 1999. He was a director of Computer Center, National Central University from 2003 to 2006. His current research interests include wireless communications and mobile computing. He has been an associate editor of the IEEE Transactions on Parallel and Distributed Systems and is an associate editor of the International Journal of Ad Hoc and Ubiquitous Computing and International Journal of Sensor Networks. He received Distinguished Research Awards from the National Science Council of the Republic of China in 1993-1994, 1995-1996, and 1997-1998, the Distinguished Engineering Professor Award from the Chinese Institute of Engineers in 2003, the K.-T. Li Research Breakthrough Award of the Institute of Information and Computing Machinery in 2007 and the Y.Z. Hsu Scientific Chair Professor Award in 2009. He is a fellow of the IEEE, a member of the ACM, and the Phi Tau Phi Society.

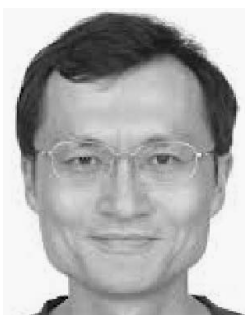

Chung-Ta King received the BS degree in electrical engineering from National Taiwan University, R.O.C., in 1980, and the MS and $\mathrm{PhD}$ degrees in computer science from Michigan State University, East Lansing, in 1985 and 1988, respectively. From 1988 to 1990 , he was an assistant professor of computer and information science at the New Jersey Institute of Technology. In 1990, he joined the faculty of the Department of Computer Science, National Tsing Hua University, Taiwan, where he is currently a professor. His research interests include parallel and distributed processing, networked embedded systems, and computer architecture.

$\triangleright$ For more information on this or any other computing topic, please visit our Digital Library at www.computer.org/publications/dlib. 